\title{
COEFFICIENTS DE FOURIER DE FONCTIONS À VARIATION BORNÉE
}

\author{
JIE WU
}

(Communicated by J. Marshall Ash)

\begin{abstract}
Let $f: \mathbb{R} \rightarrow \mathbb{C}$ be a function of period $2 \pi$ and of bounded variation on $[0,2 \pi]$ with the total variation $V(f)$. Its Fourier coefficients are denoted by $\hat{f}(n)$. In this short note, we give a very simple proof of the known result $|\hat{f}(n)| \leq V(f) / 2 \pi|n| \quad(n \in \mathbb{Z}, n \neq 0)$.
\end{abstract}

Soit $f(t)$ une fonction définie sur $\mathbb{R}$, à valeurs dans $\mathbb{C}$, de période $2 \pi$, et à variation bornée sur $[0,2 \pi]$. On désigne respectivement par $V(f)$ et $\hat{f}(n)$ sa variation totale sur $[0,2 \pi]$ et ses coefficients de Fourier:

$$
\hat{f}(n)=\frac{1}{2 \pi} \int_{0}^{2 \pi} f(t) e^{-i n t} d t \quad(n \in \mathbb{Z}, n \neq 0) .
$$

A l'aide de la théorie de l'intégration de Riemann-Stieltjies, on peut facilement démontrer que

$$
|\hat{f}(n)| \leq V(f) / 2 \pi|n| \quad(n \in \mathbb{Z}, n \neq 0),
$$

et cette majoration est optimale (voir [2]). Il est intéressant de chercher une démonstration de (1) sans utiliser la notion de l'intégration de Riemann-Stieltjes. Dans cette direction, Taibleson [3] a démontré, par une méthode très élégante, que

$$
|\hat{f}(n)| \leq V(f) /|n| \quad(n \in \mathbb{Z}, n \neq 0) .
$$

La démonstration de Taibleson est citée intégralement par Edwards dans son livre [1]. Pourtant (2) est moins précis que (1), d'un facteur multiplicatif $1 / 2 \pi$. Edwards [1] a donné une démonstration élémentaire de (1), sans utiliser la notion de l'intégration de Riemann-Stieltjes, mais sa méthode est plus compliquée que celle de Taibleson. Dans cette note courte, nous modifions la méthode de Taibleson et donnons une démonstration très simple de (1).

Soient $N, n \in \mathbb{Z}$ avec $N>0, n \neq 0$. On pose

$$
a_{k}=2 \pi k / N|n| \quad(k=0,1, \ldots, N|n|) .
$$

Received by the editors June 20, 1991.

1991 Mathematics Subject Classification. Primary 42A16.

Key words and phrases. Fourier coefficients. 
Désignons par $V_{k}(f)$ la variation totale de $f$ sur $\left[a_{k-1}, a_{k}\right]$. Nous écrivons d'abord une suite d'inégalités

$$
\begin{aligned}
2 \pi|\hat{f}(n)| & \leq\left|\sum_{k=1}^{N|n|} f\left(a_{k}\right) \int_{a_{k-1}}^{a_{k}} e^{-i n t} d t\right|+\left|\sum_{k=1}^{N|n|} \int_{a_{k-1}}^{a_{k}}\left(f(t)-f\left(a_{k}\right)\right) e^{-i n t} d t\right| \\
& \leq \frac{1}{|n|}\left|\sum_{k=1}^{N|n|} f\left(a_{k}\right)\left(e^{-i n a_{k-1}}-e^{-i n a_{k}}\right)\right|+\sum_{k=1}^{N|n|} V_{k}(f)\left(a_{k}-a_{k-1}\right) .
\end{aligned}
$$

Ensuite, en remarquant les faits suivants

$$
\begin{array}{cc}
f\left(a_{0}\right)=f\left(a_{N|n|}\right), & \sum_{k=1}^{N|n|} V_{k}(f) \leq V(f), \\
a_{k}-a_{k-1}=\frac{2 \pi}{N|n|} \quad(1 \leq k \leq N|n|),
\end{array}
$$

on peut déduire

$$
\begin{aligned}
2 \pi|\hat{f}(n)| & \leq \frac{1}{|n|}\left|\sum_{k=1}^{N|n|}\left(f\left(a_{k}\right)-f\left(a_{k-1}\right)\right) e^{-i n a_{k-1}}\right|+\frac{2 \pi V(f)}{N|n|} \\
& \leq\left(1+\frac{2 \pi}{N}\right) \frac{V(f)}{|n|} .
\end{aligned}
$$

Finalement en faisant $N$ tendre vers $+\infty$, nous obtenons

$$
|\hat{f}(n)| \leq V(f) / 2 \pi|n| \quad(n \in \mathbb{Z}, n \neq 0),
$$

comme souhaité.

\section{BIBLIOGRAPHIE}

1. R. E. Edwards, Fourier Series, Vol. 1, Springer-Verlag, New York and Heidelberg, 1979.

2. G. H. Hardy and W. Rogosinski, Fourier series, Cambridge Univ. Press, New York, 1965.

3. M. Taibleson, Fourier coefficients of functions of bounded variation, Proc. Amer. Math. Soc. 18 (1967), 766.

Departement de MathématiQues, Unite associée au CNRS, URA 750, Université de NANCY I, 54506 VANDGeUvRe-LES-NANCY, FRANCE

E-mail address:wujie@iecn.u-nancy.fr 